Cite as: Stephane H Maes, (2020), "Massless and Massive Multi-Gravity in a Multi-fold Universe", viXra:2010.0095v1, https://vixra.org/pdf/2010.0095v1.pdf, https://shmaesphysics.wordpress.com/2020/06/30/ massless-and-massive-multi-gravity-in-a-multi-fold-universe/, June 19, 2020.

\title{
Massless and Massive Multi-Gravity in a Multi-fold Universe
}

\author{
Stephane H. Maes ${ }^{1}$
}

June 30, 2020

\begin{abstract}
:
In a multi-fold universe, gravity emerges from Entanglement through the multi-fold mechanisms. As a result, gravity-like effects appear in between entangled particles that they be real or virtual. Long range, massless gravity results from entanglement of massless virtual particles. Entanglement of massive virtual particles leads to massive gravity contributions at very smalls scales. Multi-folds mechanisms also result into a spacetime that is discrete, with a random walk fractal structure and non-commutative geometry that is Lorentz invariant and where spacetime nodes and particles can be modeled with microscopic black holes. All these recover General relativity at large scales and semi-classical model remain valid till smaller scale than usually expected. Gravity can therefore be added to the Standard Model. This can contribute to resolving several open issues with the Standard Model.

In this paper, we discuss some of the properties and implications of the massive gravity contributions. In particular we will discuss it similarities and differences with what is usually meant by massive gravity in conventional physics and derived modern bigravity theories.. Instead of being a large-scale theory, where massive gravity can support a limited range or even a repulsive behavior, multi-fold massive gravity is here a mostly small-scale effect with almost no larger scale impact other than through entangled virtual neutrino pairs. Multi-fold universe accelerated expansion come from other effects of multi-fold mechanisms. In multi-fold theory, massive gravity is also multiple (one per available virtual carrier). The resulting gravity model is different from all the massive gravity and bigravity current proposed in the literature. In particular we discuss the known issues with conventional classical massive gravity.
\end{abstract}

We conclude with a suggestion to attempt, with or independently of a multi-fold models, bi (or multi) gravity models, massive only at very small scale with massless gravity at any larger scale. We already know that such model helps address many Standard Model and Standard Cosmology Model open issues: there is value in SMG: the standard Model with non-negligible gravity at its scales.

\section{Introduction}

The new preprint [1] proposes contributions to several open problems in physics like the reconciliation of General Relativity (GR) with Quantum Physics, explaining the origin of gravity proposed as emerging from quantum (EPREinstein Podolsky Rosen) entanglement between particles, detailing contributions to dark matter and dark energy and explaining other Standard Model mysteries without requiring New Physics beyond the Standard Model other than the addition of gravity to the Standard Model Lagrangian. All this is achieved in a multi-fold universe that may well model our real universe, which remains to be validated.

With the proposed model of [1], spacetime and Physics are modeled from Planck scales to quantum and macroscopic scales and semi classical approaches appear valid till very small scales. In [1], it is argued that spacetime is discrete, with a random walk-based fractal structure, fractional and noncommutative at, and above

\footnotetext{
${ }^{1}$ shmaes.physics@gmail.com
} 
Planck scales (with a 2-D behavior and Lorentz invariance preserved by random walks till the early moments of the universe). Spacetime results from past random walks of particles. Spacetime locations and particles can be modeled as microscopic blackholes (Schwarzschild for photons and spacetime coordinates, and metrics between Reisner Nordstrom [2] and Kerr Newman [3] for massive and possibly charged particles - the latter being possibly extremal). Although surprising, [1] recovers results consistent with other like [4], while also being able to justify the initial assumptions of black holes from the gravity or entanglement model in a multi-fold universe. The resulting gravity model recovers General Relativity at larger scale, as a 4-D process, with massless gravity, but also with massive gravity components at very small scale that make gravity significant at these scales. Semi-classical models also turn out to work well till way smaller scales that usually expected.

In this paper, we remain at a high level of discussion of the analysis and references are generic for the subjects. It makes the points accessible to a wider audience and keeps the door open to further papers or discussions devoted to details of interest. Yet, it requires the reader to review [1], as we do not revisit here all the details of the multifold mechanisms or reconstruction of spacetime. More targeted references for all the material discussed here are compiled in [1].

\section{Conventional Massive Gravity: Problems and Breakthroughs}

Massive gravity has been around since the work of [5] for a spin-2 massive (linear) gravity. An historical overview, with reference as well as detailed reviews, that also fairly well explain the assertions in [6], can be found respectively in $[6]$ and $[7,8]$. It is far to say that massive gravity has presented multiple challenges related to and the presence of discontinuities between massive gravity models and massless models due to non-vanishing extra degrees of freedom, the need for suitable non-linearities themselves to address the discontinuity that themselves introduce ghosts and other schemes like screening effects which are unstable or divergent, supra luminosity and acausality. Then [9], introduced a solution that is ghost free: the dRGT massive gravity. It also can fit cosmological models like $\Lambda$-CDM [10], the Standard Cosmological Model [27,32]. [28,29] provided for stabler massive gravity model. While argument still exist (e.g. [30,31]) that supra luminosity or acausality is encountered, ([7]-section 10.6) argues very convincingly again taking them as serious issues mainly because associated to non-physical effects or beyond the limits of validity of the classical models.

It seems logical to also try combining massive and massless gravity. It is known as bigravity or partially massless gravity $[10,7]$. Again different approaches exist and its latest incarnation as combining the Hilbert Einstein action with the dRGT Action (both with weight factors that amount to different Planck constants for each contribution). One can see how this could also evolve into a multi-gravity, but to our knowledge that has not been that seriously considered.

On one hand, bi or multi-gravity can depend on massless gravity or only add to it . So for example, in curved space (e.g. curved by massless effects), ghosts also disappear [8].

Yet all these approaches affect the long ranges and with very small mass graviton, constrained by $\Lambda-C D M$ and gravitation wave observation (if/when detected hand in hand with electromagnetic events, as was just claimed recently, these boundaries will become stricter or massive gravity effects will be disproved or validated. As far as we have seen so far, no new claims have been made yet) and many still have discrepancies with some of the cosmology observations. They try to have no observable effects at smaller scales so that hey do not affect how conventional massless gravity works.

The most important observation from this section is that it is possible to formulate a reasonable, ghost-free massive theory of gravity or bigravity and even argue for stability, no supra luminosity and no acausality. Renormalization may not be an issue [7] in massive some gravity [8]. Remember that conventional massless (quantum)gravity is not renormalizable so far [12]. All these points are good news for the multi-fold universes which include massive / bi / multi gravity [1], and for which we know that the massless gravity contribution can recover $\mathrm{GR}$ at large scales. 


\section{Gravity in Multi-fold Universes}

We refer to [1] for the details of the model. The important observations are:

- Multi-fold mechanisms introduced to address the EPR paradox, result into gravity like attractive potential in spacetime between the entangled particles (See also [13]).

- If entangled virtual particles emitted by a sources are considered as the source of gravity, then entangled virtual photons result into a massless gravity contribution that recovers $G R$ at larger scales.

- Virtual neutrino pairs where not really distinguished because of their small mass and large speed; but rigorously they are a first long-range massive gravity contribution certainly fitting not impacting local observations (to be seen in a multi-fold universe as bi-gravity due respectively to entangled virtual pairs of photons and neutrinos).

- Most of the other virtual pairs (fermions and Bosons) are massive (or contained) and therefore offer massive gravity at small scales; not large scales. They have very small ranges dictated by their masses.

- Entanglement adds gravity like contributions [13], that could explain dark matter [14].

Therefore, in a multi-fold universe, we have a large scale bi-gravity that may be modeled by bi-gravity at large scale and multi-gravity at small scales; it is a multi-gravity [7]. The massless part (or massless = massive within the range of the neutrinos) can recovers $G R$.

The VDVZ discontinuity [6], whereby massive gravity does not converge to massless gravity when the mass of the graviton goes to zero can be understood in a multi-fold universe as follow:

- Small range effects can't converge to large range effects: the limit makes no sense or said differently the discontinuity makes a lot of sense.

- Only entanglement of neutrinos virtual pairs can be seen as a long-range massive gravity contribution. Again a limit to zero does not make sense: it is or it is not associated to massless neutrinos. As neutrinos are not massless, the convergence is not meaningful.

- The notion of massive graviton vs. massless is not really something that makes sense. They are realization of the multi-fold dynamics [1] and behaviors of the associated entangled virtual particles. In between particles types, the notion of limit is meaningless.

\section{Small and Very Small Scales Implications}

(Sorry for the self-citations)

As result, at small scale, gravity is no more negligible and we recommend adding its effect to the Standard Model. This resulted into providing solutions to several Standard model open issues without New Physics other than this addition of gravity $\left(\mathrm{SM}_{\mathrm{G}}\right)[1]$ :

- Why no proton decays [15]?

- Why no magnetic monopoles [16]?

- Why 3 and only 3 generations of fermions per family [17]?

- Why no strong CP violation [18]?

- Where can the neutrino masses come from and where did the right-handed neutrino go [19]?

- Why the Yang Mills Mass gap problem may not be already resolved [20]?

- Why is the Electroweak vacuum actually stable, despite the mass of the Higgs boson [21]?

As well as explaining cosmological problem again without adding new physics to the Standard Model / Standard Cosmological Model other than our multi-fold mechanisms and $\mathrm{SM}_{\mathrm{G}}$ [1]:

- Accelerated expansion, Dark Energy and small cosmological constant [11]

- Inflation with or without inflaton [11] 


\section{- $\quad$ Dark Matter [14]}

Eventually, it resulted into revisiting the Weak Gravity Conjecture (WCG) [22] (no more valid at small scales in a multi-fold universe) and a new proposal for force unification: the Unified Unifications (UU) [23]. Based on the new analysis à la WCG [22], we see that all interactions equally contribute to gravity and their proper interactions at very small scales, resulting into a democracy of effects: a different twist on Grand Unification.

Because spacetime in [1] is discrete, fractal, noncommutative and Lorentz invariant, we can ensure (re)normalization of gravity. In addition to discreteness, torsion and dark energy / random walk effects ensure the absence of gravity (and cosmological singularities).

See [33] to follow-up the latest updates and new results related to multi-fold universe. Many illustrate the importance of SMG for SM and the Standard Cosmological Model.

\section{Discussion}

In our multi-fold universe, the Lagrangian (density) contribution from a massive component gravity relates to the massless gravity by:

$$
\mathscr{L}_{\text {massless }}\left(G_{m}\right)=\mathscr{L}_{\text {massive }}\left(G_{m}\right)+\Delta\left(G_{m}\right)
$$

Where $\quad G_{m}$. designates the massive gravity constant and usage of metric associated to it. $\Delta\left(G_{m}\right)$ designates the potential energy by not fully extending gravity.

If we repeat the GR recovery arguments of [1], with regions where massive gravity does not reach, we recover field equation that correspond to (1) with $\Delta\left(G_{m}\right)$ encountered as all the missing contributions tapered or missing due to the limited reach.

Bigravity similarly is expressed as:

$$
\mathscr{L}_{B i}\left(G_{m}, G_{0}\right)=\mathscr{L}_{\text {massless }}\left(G_{0}\right)+\mathscr{L}_{\text {massless }}\left(G_{m}\right)-\Delta\left(G_{m}\right) \text { (2) }
$$

Where $G_{0}$ correspond to the massless gravity constant. Multi gravity is similarly handled [7].

These equation match [7] (equations 6.6 - 6.7). So we know that in a multi-fold universe, the massive / bi or multi gravity terms can correspond to the terms of dRGT. We also know that there are no one but many dRGT solutions. We recover one of them as we are by construction Ghost free and renormalized. Finding concrete version of the recovered dRGT gravity does not seem immediate though.

The multi gravity equation version can be thought as the GR (i.e. classical / curvature based) version of the gravity discovered in multi-fold universe where $\mathscr{L}_{\text {massless }}\left(G_{0}\right)$ is the conventional Hilbert Einstein Action.

\section{Closing The Open Issues With Massive Gravity and Some}

\section{Consequences}

Based on the derivation in [1] (multi-fold and reconstruction phase), we encounter massive gravity at small scale in addition to massless. No Ghost are encountered. Which lead us to the conclusion that classic approximation must either contain no Ghost or reject them as unphysical because of limitation of the model. Indeed we clearly see the 
quantum aspects of massive gravity to consider: i) very small and small scales ii) carried by massive virtual particles, a quantum only effect iii) resulting from entanglement.

As a result, the arguments of ([7]-section 10.6) against taking supra luminous effects and acausality as serious issues in classical approximations fully hold. Indeed in a multi-fold universe, by reconstruction, spacetime is discrete, fractal (by random walks), Lorentz invariant and non-commutative at very small scales. This ensures that supra luminosity will not take place and that acausality is not occurring.

In multi-fold universe, these effects in classical model are unphysical and do not impact the suitability of proposing a small-scale massive gravity contribution; especially as it is so important to justify the introduction $\mathrm{SM}_{\mathrm{G}}$.

At large scale, we have essentially ignored the mass of the neutrinos. We know it is non-zero, and so there will be observable effects (e.g. echoes in gravitational waves and echoes or delays in multi-messenger astronomical observations). Yet today this is not detectable. Also because neutrinos are of such a small mass and virtual neutrino pairs emissions compete with virtual photon pair emissions, which are more probable, we do expect that besides being hard to observe, the effect is very small at large scales and mostly results into echoes rather than modifications of gravity at large range as would be proposed with MOND inspired approaches [34] (or conventional expectations for massive gravity where the mass is expected to play a larger role). It is however also a prediction of our model and such large-scale effects exists and should be detectable at some point and another possible way to validate our multi-fold theory. Small-scales effect also of course, but this is probably even harder to detect anytime soon.

\section{Conclusions}

This analysis is for a Multi-fold universe as in [1]. [1] details arguments and ways to check its relationship with the real universe. Besides properties that can be experimentally verified (in the future because of the macroscopic weakness of gravity and gravity like effects for entangled systems as well as small-scale effect and corroboration of the value of considering $\mathrm{SM}_{\mathrm{G}}$ ), [1] shows how the multi-fold mechanisms and behaviors are in many aspects in today's conventional physics, that, at times, anticipate the behaviors modeled in a multi-fold universe. In addition, $[1,33]$ potentially explains many results obtained in gravity, quantum mechanics, General Relativity, superstring theory, Loop Quantum Gravity and the AdS/CFT correspondence conjecture. All these works attempt to come up with models for the real universe. It is at least a good sign that [1] may provide an interesting model of the real universe.

We believe that [1] makes a compelling case for the consistency of its multi-fold proposal. Part of our analysis has no equivalent or variations for non multi-fold universe (e.g. dark matter explanation or gravity like effects due to entanglement). Yet modeling a multi gravity solution with large effects at small and very small ranges and bigravity at very large range (or just $\sim$ massless if both components are essentially combined / or if virtual neutrino pairs are not significant (something that multi-messenger astronomy could maybe help determine [24]) could be of great interest. We hope that it may be of interest to massive gravity experts. From our point of view, we would like to see what actual action (i.e. $\Delta\left(G_{m}\right) \quad$ ) and other terms would be recovered when we try to repeat GR recovery for the massive contributions. Doing so if for future works but plausible when quickly reasoning as in [1], as semiclassical models can apply, in multi-fold universes, to way smaller ranges than probably expected.

Note (10/5/20): By having the multi-fold (and graviton) evolving in AdS(5), we escape any issue of spin-2 massive terms (e.g. no-go theorem if it were applicable as discussed in [25] that seems proven avoidable anyway [9,25]). Massive gravity is also not to affected by the Weinberg-Witten no-go theorem [26]). We have no particular constraint imposed on the multi-folds associated to massive or massless behaviors. 
References: (most references come from popular science to make the discussion more approachable)

[1]: Stephane H. Maes, (2020) "Quantum Gravity Emergence from Entanglement in a Multi-Fold

Universe", viXra:2006.0088v1, (June 9, 2020).

[2]: https://en.wikipedia.org/wiki/Reissner\%E2\%80\%93Nordstr\%C3\%B6m metric

[3]: https://en.wikipedia.org/wiki/Kerr-Newman metric

[4]: Burinskii, Alexander, (2008), "The Dirac-Kerr-Newman electron", arXiv:0507109v4

[5]: M. Fierz, Wolfgang Ernst Pauli, (1939), "On relativistic wave equations for particles of arbitrary spin in an

electromagnetic field", Proc. R. Soc. Lond. A173211-232

[6]: https://en.wikipedia.org/wiki/Massive gravity

[7]: Claudia de Rham, (2014), "Massive Gravity", arXiv:1401.4173v2

[8]: Kurt Hinterbichler, (2011), "Theoretical Aspects of Massive Gravity", arXiv:1105.3735v2

[9]: Claudia de Rham, Gregory Gabadadze, Andrew J. Tolley, (2010), "Resummation of Massive Gravity", arXiv:1011.1232v2

[10]: https://en.wikipedia.org/wiki/Lambda-CDM model

[11]: https://en.wikipedia.org/wiki/Bimetric gravity

[12]: Assaf Shomer (2007). "A pedagogical explanation for the non-renormalizability of gravity", arXiv:0709.3555v2

[13]: Stephane H Maes, (2020), "Gravity-like Attractions and Fluctuations between Entangled

Systems?", viXra:2010.0010v1, https://shmaesphysics.wordpress.com/2020/06/25/gravity-like-attractions-and-

fluctuations-between-entangled-systems/, June 24, 2020.

[14]: Stephane H Maes, (2020), "Explaining Dark Matter Without New

Physics?", viXra:2006.0088v1, https://shmaesphysics.wordpress.com/2020/06/19/explaining-dark-energy-smallcosmological-constant-and-inflation-without-new-physics/, June 21, 2020.

[15]: Stephane H Maes, (2020), "Gravity Induced Anomalies Smearing in Standard Model so that Protons May Never Decay, Except in Black

holes", viXra:2006.0128v1, https://shmaesphysics.wordpress.com/2020/06/12/protons-may-never-decay-exceptin-black-holes/, June 13, 2020.

[16]: Stephane H Maes, (2020), "Gravity or Magnetic Monopoles? You Cannot Have

Both!", viXra:2006.0190v1, https://shmaesphysics.wordpress.com/2020/06/15/gravity-or-magnetic-monopoles-

you-cannot-have-both/, June 15, 2020.

[17]: Stephane H Maes, (2020), "Gravity Dictates the Number of Fermion Generations:

3", viXra:2007.0068v1, https://shmaesphysics.wordpress.com/2020/06/24/gravity-dictates-the-number-offermion-generations-3/, June 24, 2020.

[18]: Stephane H Maes, (2020), "Strong CP Violation Tamed in The Presence of Gravity", viXra:2007.0025v1, https://shmaesphysics.wordpress.com/2020/06/23/strong-cp-violation-tamed-in-the-presence-of-gravity/, June 21, 2020.

[19]: Stephane H Maes, (2020), "Right-handed neutrinos? Mass? Ask

Gravity", viXra:2007.0018v1, https://shmaesphysics.wordpress.com/2020/06/21/right-handed-neutrinos-askgravity/, June 21, 2020.

[20]: Stephane H Maes, (2020), " Progress on Proving the Mass gap for Yang Mills and Gravity (maybe it's already proved...)", viXra:2006.0155v1, https://shmaesphysics.wordpress.com/2020/06/12/progresses-on-proving-themass-gap-for-yang-mills-and-gravity-maybe-its-already-proven/, June 12, 2020.

[21]: Stephane H Maes, (2020), "Gravity Stabilizes Electroweak Vacuum - No Bubble of Nothing to Worry About!", viXra:2007.0173v1, https://shmaesphysics.wordpress.com/2020/06/24/gravity-stabilizes-electroweak-vacuum-nobubble-of-nothing-to-worry-about/, June 24, 2020.

[22]: Nima Arkani-Hamed, Lubos Motl, Alberto Nicolis, Cumrun Vafa, (2006), “The String Landscape, Black Holes and Gravity as the Weakest Force", arXiv:hep-th/0601001v2.

[23]: Stephane H Maes, (2020), "Ultimate Unification: Gravity-led Democracy vs. Uber-

Symmetries", viXra:2006.0211v1, https://shmaesphysics.wordpress.com/2020/06/16/ultimate-unification-gravityled-democracy-vs-uber-symmetries/, June 16, 2020.

[24]: Marica Branchesi, (2016), "Multi-messenger astronomy: gravitational waves, neutrinos, photons, and cosmic rays", J. Phys.: Conf. Ser. 718022004

[25]: Sarah Folkerts, Cristiano Germani, Nico Wintergerst, (2013), "Massive spin-2 theories", arXiv:1310.0453v2 
[26]: https://en.wikipedia.org/wiki/Weinberg\%E2\%80\%93Witten theorem

[27]: Claudia de Rham, Lavinia Heisenberg, Raquel H. Ribeiro, (2013), "Quantum Corrections in Massive Gravity", arXiv:1307.7169v2

[28]: S. F. Hassan, Rachel A. Rosen, (2011), "On Non-Linear Actions for Massive Gravity", arXiv:1103.6055v3

[29\}: S. F. Hassan, Rachel A. Rosen, (2012), " Resolving the Ghost Problem in non-Linear Massive Gravity", arXiv:1106.3344v3

[30]: Andrei Gruzinov, (2011), "All Fierz-Paulian massive gravity theories have ghosts or superluminal modes", arXiv:1106.3972v1

[31]: S. Deser, K. Izumi, Y.C. Ong, A.Waldron, (2013), "Massive Gravity Acausality Redux", arXiv:1306.5457v3

[32]: Yashar Akrami, Tomi S. Koivisto, Marit Sandstad, (2012), "Accelerated expansion from ghost-free bigravity: a statistical analysis with improved generality", arXiv:1209.0457v3

[33]: Stephane H. Maes, (2020), "Web Site Tracking all Publications around the Multi-fold universe", Navigation page listing all papers. https://shmaesphysics.wordpress.com/shmaes-physics-site-navigation/

[34]: Wikipedia, "Modified Newtonian dynamics", https://en.wikipedia.org/wiki/Modified Newtonian dynamics. Retrieved in March 2019. 